\title{
Determining the Optimal Decision Delay Parameter for a Linear Equalizer
}

\author{
Eng Siong Chng \\ School of Computer Engineering, Nanyang Technological University, Nanyang Avenue, Singapore 639798. \\ Sheng Chen* \\ School of Electronics and Computer Science, University of Southampton, Highfield Southampton SO17 1BJ, U.K.
}

\begin{abstract}
The achievable bit error rate of a linear equalizer is crucially determined by the choice of a decision delay parameter. This brief paper presents a simple method for the efficient determination of the optimal decision delay parameter that results in the best bit error rate performance for a linear equalizer.
\end{abstract}

Keywords: Linear equalizer, decision delay, bit error rate.

\section{Introduction}

Equalization techniques play an ever-increasing role in combating distortion and interference in modern communication links ${ }^{[1,2]}$. It is well-known that the choice of equalizer decision delay parameter critically determines achievable bit error rate (BER) performance $^{[3,4]}$. We present a simple and effective method for determining an optimal decision delay parameter that results in the best bit error rate performance for a linear equalizer. The proposed technique computes a relative measure for each decision delay value that characterizes the degree of linear separability between the different signal classes for the given decision delay value. From the resulting set of measures, for every decision delay value, it is straightforward to choose the optimal decision delay that provides the best achievable BER performance.

Consider the baseband digital communication system depicted in Fig.1. The received signal, after the communication channel, sampled at a symbol rate, is modeled by ${ }^{[1,2]}$

$$
\boldsymbol{x}(k)=\sum_{i=0}^{M} h_{i} s(k-i)+n(k)
$$

where $k$ denotes the symbol index, $n(k)$ is white Gaussian noise with variance $\sigma_{n}^{2}, h_{i}$ are the taps of the channel impulse response (CIR) which has a memory $M$, and $s(k)$ is a binary input drawn from the set $\{ \pm 1\}$ with equal probability. Although the analysis presented here assumes binary phase shift keying (BPSK) modulation, the results can be generalized.

Manuscript received November 26, 2003; revised September 2, 2004.

*Corresponding author. E-mail address: sqc@ecs.soton.ac.uk

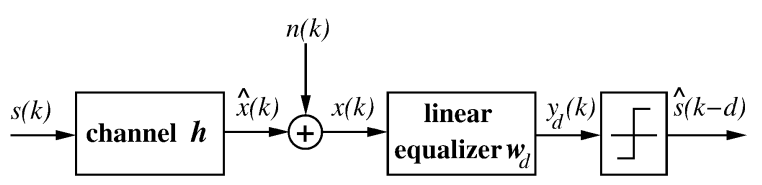

Fig.1 Baseband model of a communication system with a linear equalizer

A linear equalizer with length $N$ and decision delay $d$ uses a vector of noisy observations

$$
\boldsymbol{x}(k)=[x(k) x(k-1) \cdots x(k-N+1)]^{T}
$$

to provide an estimate $\hat{s}(k-d)$ of the transmitted symbol $s(k-d)$, and is specified by

$$
y_{d}(k)=\boldsymbol{w}_{d}^{T} \boldsymbol{x}(k)=\sum_{i=0}^{N-1} w_{d_{i}} x(k-i)
$$

and

$$
\hat{s}(k-d)=\operatorname{sgn}\left(y_{d}(k)\right)
$$

where $\operatorname{sgn}(\bullet)$ denotes the sign function and

$$
\boldsymbol{w}_{d}=\left[w_{d_{0}} w_{d_{1}} \cdots w_{d_{N-1}}\right]^{T}
$$

is an equalizer weight coefficient vector.

An equalizer input vector $x(k)$ is given by

$$
\boldsymbol{x}(k)=H \boldsymbol{s}(k)+\boldsymbol{n}(k)=\overline{\boldsymbol{x}}(k)+\boldsymbol{n}(k)
$$

where $s(k)=[s(k) s(k-1) \cdots s(k-L+1)]^{T}$ is a vector of $L=M+N$ transmitted digital symbols, $\boldsymbol{n}(k)=[n(k) n(k-1) \cdots n(k-N+1)]^{T}$ is a noise vector, $\overline{\boldsymbol{x}}(k)$ is a vector of noise-free input signal called the channel state, and $H$ is a $N \times L$ channel convolution matrix given by

$$
H=\left[\begin{array}{ccccccc}
h_{0} & h_{1} & \cdots & h_{M} & 0 & \cdots & 0 \\
0 & h_{0} & h_{1} & \cdots & h_{M} & \ddots & \vdots \\
\vdots & \ddots & \ddots & \ddots & \ddots & \ddots & 0 \\
0 & \cdots & 0 & h_{0} & h_{1} & \cdots & h_{M}
\end{array}\right]
$$


It is obvious that $\boldsymbol{x}(k)$ depends only on $L$ symbols in $\boldsymbol{s}(k)$ and hence the valid range of decision delay is

$$
d \in \mathcal{D}=\{0,1, \cdots, L-1\} .
$$

Given a decision delay $d$, various designs for the equalizer weight vector $\boldsymbol{w}_{d}$ can be considered. The best known design is the minimum mean square error (MMSE) solution $\hat{\boldsymbol{w}}_{d}$, which minimizes the mean square error $E\left[\left(s(k-d)-y_{d}(k)\right)^{2}\right]$ and is given by ${ }^{[1,2]}$

$$
\hat{\boldsymbol{w}}_{d}=\left(H H^{T}+\sigma_{n}^{2} \boldsymbol{I}_{N}\right)^{-1} h_{d}
$$

where $\boldsymbol{h}_{d}$ is the $(d+1)$ th column of $H$ and $\boldsymbol{I}_{N}$ denotes an $N \times N$ identity matrix. Alternatively, the minimum BER (MBER) solution $\tilde{\boldsymbol{w}}_{d}$ is obtained by directly minimizing the BER $P_{E}\left(\boldsymbol{w}_{d}\right)$ of an equalizer with decision delay $d^{[5 \sim 7]}$. The main purpose of this brief paper is to determine the optimal decision delay for the given weight vector design that achieves the smallest BER.

\section{The bit error rate}

By denoting $N_{s}=2^{L}$ combinations of $\boldsymbol{s}(k)$ as $\boldsymbol{s}_{i}$, $1 \leqslant i \leqslant N_{s}$, i.e.

$$
\boldsymbol{s}(k) \in \mathcal{S}=\left\{\boldsymbol{s}_{i}, 1 \leqslant i \leqslant N_{s}\right\}
$$

then $\overline{\boldsymbol{x}}(k)$ only takes values from the channel state set defined by

$$
\overline{\boldsymbol{x}}(k) \in \mathcal{X}=\left\{\overline{\boldsymbol{x}}_{i}=H \boldsymbol{s}_{i}, 1 \leqslant i \leqslant N_{s}\right\} .
$$

The set of channel states $\mathcal{X}$ can be divided into two subsets conditioned on the value of $s(k-d)$. Specifically, by denoting the $d$ th element of $s_{i}$ as $s_{d, i}$. Then $s_{d, i}$ specifies which class $(+1$ or -1$) \overline{\boldsymbol{x}}_{i}$ belongs to.

Given the equalizer's weight vector $\boldsymbol{w}_{d}$ for a fixed decision delay $d$, by defining the normalized decision variable for $\overline{\boldsymbol{x}}_{i}$ as

$$
\zeta_{i}\left(\boldsymbol{w}_{d}\right)=\frac{\boldsymbol{w}_{d}^{T} \overline{\boldsymbol{x}}_{i}}{\left\|\boldsymbol{w}_{d}\right\|}
$$

where $\|\boldsymbol{w}\|=\sqrt{\boldsymbol{w}^{T} \boldsymbol{w}}$. Then $\overline{\boldsymbol{x}}_{i}$ is correctly classified by $\boldsymbol{w}_{d}$ if and only if

$$
\operatorname{sgn}\left(\zeta_{i}\left(\boldsymbol{w}_{d}\right)\right)=\operatorname{sgn}\left(s_{d, i}\right) .
$$

The BER of this equalizer is evaluated by ${ }^{[6,7]}$

$$
P_{E}\left(\boldsymbol{w}_{d}\right)=\frac{1}{N_{s}} \sum_{i=1}^{N_{s}} p_{e}\left(\zeta_{i}\left(\boldsymbol{w}_{d}\right)\right)
$$

where $p_{e}\left(\zeta_{i}\left(\boldsymbol{w}_{d}\right)\right)$ denotes the probability of error due to the received channel state being $\overline{\boldsymbol{x}}_{i}$, and is evaluated by

$$
p_{e}\left(\zeta_{i}\left(\boldsymbol{w}_{d}\right)\right)=\left\{\begin{array}{l}
Q\left(\frac{\left|\zeta_{i}\left(\boldsymbol{w}_{d}\right)\right|}{\sigma_{n}}\right), \overline{\boldsymbol{x}}_{i} \text { is correctly classified } \\
1-Q\left(\frac{\left|\zeta_{i}\left(\boldsymbol{w}_{d}\right)\right|}{\sigma_{n}}\right), \text { otherwise }
\end{array}\right.
$$

where $|\bullet|$ denotes the absolute value and

$$
Q(x)=\frac{1}{\sqrt{2 \pi}} \int_{x}^{\infty} \exp \left(-\frac{v^{2}}{2}\right) d v
$$

Note that $\left|\zeta_{i}\left(\boldsymbol{w}_{d}\right)\right|$ is the distance of the channel state $\overline{\boldsymbol{x}}_{i}$ to the decision boundary specified by $\boldsymbol{w}_{d}^{T} \boldsymbol{x}=$ 0 . For $\boldsymbol{w}_{d}$ to achieve the desired linear separability, all $\overline{\boldsymbol{x}}_{i}$ must be correctly classified by $\boldsymbol{w}_{d}$, that is, condition (13) must hold for $i=1,2, \cdots, N_{s}$. Since $Q(\bullet)$ decays exponentially, BER $P_{E}\left(\boldsymbol{w}_{d}\right)$ is dominated by the largest $p_{e}\left(\zeta_{i}\left(\boldsymbol{w}_{d}\right)\right)$ when $\sigma_{n} \rightarrow 0$. Thus an upper bound of the BER is given by

$$
P_{E_{U B}}\left(\boldsymbol{w}_{d}\right)=\max _{1 \leqslant i \leqslant N_{s}}\left\{p_{e}\left(\zeta_{i}\left(\boldsymbol{w}_{d}\right)\right)\right\}
$$

\section{Optimal decision delay}

Optimal decision delay can in theory be defined by

$$
d_{o p t}=\arg \min _{d \in \mathcal{D}} P_{E_{U B}}\left(\boldsymbol{w}_{d}\right)
$$

From the definition of $p_{e}(\bullet)$ in (15), it is obvious that optimal decision delay can alternatively be determined by

$$
d_{\text {opt }}=\arg \max _{d \in \mathcal{D}}\left\{\min _{1 \leqslant i \leqslant N_{s}} \zeta_{i}\left(\boldsymbol{w}_{d}\right)\right\}
$$

To derive a computationally simpler way of evaluating $d_{\text {opt }}$, we can define

$$
\boldsymbol{f}_{d}=\left[f_{0, d} f_{1, d} \cdots f_{L-1, d}\right]^{T}=\frac{H^{T} \boldsymbol{w}_{d}}{\left\|\boldsymbol{w}_{d}\right\|}
$$

then

$$
\zeta_{i}\left(\boldsymbol{w}_{d}\right)=\frac{\boldsymbol{w}_{d}^{T} H \boldsymbol{s}_{i}}{\left\|\boldsymbol{w}_{d}\right\|}=\boldsymbol{f}_{d}^{T} \boldsymbol{s}_{i}
$$

or

$$
\zeta_{i}\left(\boldsymbol{w}_{d}\right)=f_{d, d} s_{d, i}+\sum_{\substack{j \neq d \\ j=1, \cdots, L}} f_{j, d} s_{j, i}
$$

Furthermore, $f_{d, d}$ is the main tap of the combined impulse response of the channel $\boldsymbol{h}=\left[h_{0} h_{1} \cdots h_{M}\right]^{T}$ and the normalized equalizer weight vector $\boldsymbol{w}_{d} /\left\|\boldsymbol{w}_{d}\right\|$, and $f_{d, d}>0$.

For the correct classification (13) to hold, shows that it is sufficient to have

$$
f_{d, d}+\sum_{\substack{j \neq d \\ j=1, \cdots, L}} f_{j, d} s_{j, i} s_{d, i}>0 .
$$


Taking into account $s_{j, i} \in\{ \pm 1\}$, for (13) to hold for all $\overline{\boldsymbol{x}}_{i}$, it is sufficient that

$$
\left|f_{d, d}\right|>\sum_{\substack{j \neq d \\ j=1, \cdots, L}}\left|f_{j, d}\right| .
$$

In fact, the minimum of $\left\{\zeta_{i}\left(\boldsymbol{w}_{d}\right)\right\}_{i=1}^{N_{s}}$ for decision delay $d$ is evaluated as

$$
\lambda\left(\boldsymbol{w}_{d}\right)=\min _{1 \leqslant i \leqslant N_{s}} \zeta_{i}\left(\boldsymbol{w}_{d}\right)=\left(\left|f_{d, d}\right|-\sum_{\substack{j \neq d \\ j=1, \cdots, L}}\left|f_{j, d}\right|\right) .
$$

The minimum distance measure $\lambda\left(\boldsymbol{w}_{d}\right)$ has a clear geometric interpretation. A positive $\lambda\left(\boldsymbol{w}_{d}\right)$ indicates that $\boldsymbol{w}_{d}$ achieves linear separability and a negative value otherwise. Moreover, $\lambda\left(\boldsymbol{w}_{d}\right)$ measures the relative degree of linear separability quantitatively. A negative $\lambda\left(\boldsymbol{w}_{d}\right)$ with a larger magnitude means that nonlinear separability is more severe, and a larger positive value of $\lambda\left(\boldsymbol{w}_{d}\right)$ indicates that the channel states are located further away from the linear decision boundary, which implies a better BER performance. From (19), optimal decision delay is determined as

$$
d_{\text {opt }}=\arg \max _{d \in \mathcal{D}}\left\{\lambda\left(\boldsymbol{w}_{d}\right)\right\}
$$

\section{Examples}

Example 1. The transfer function of a CIR was defined by $H_{1}(z)=0.66+1.0 z^{-1}-0.66 z^{-2}$ and equalizer length given by $N=5$. The range of decision delays was therefore $\mathcal{D}=\{0,1, \cdots, 6\}$. With a channel signal to noise ratio (SNR) of $25 \mathrm{~dB}$, the MMSE weight vectors $\hat{\boldsymbol{w}}_{d}(9)$ for each decision delay $d \in \mathcal{D}$ were calculated and the corresponding minimum distance measures $\lambda\left(\hat{\boldsymbol{w}}_{d}\right)$ were evaluated using (25). The computed $\lambda\left(\hat{\boldsymbol{w}}_{d}\right)$ for $d=0,1, \cdots, 6$ were

$$
-1.263,0.338,0.799,0.879,0.799,0.338,-1.263 \text {. }
$$

The above results indicate that $d=0$ or $d=6$ results in a nonlinearly separable equalization problem with the worst BER performance, while the best BER performance is achieved with an optimal decision delay $d=3$. To verify these predictions, simulation was conducted to evaluate the BERs of a linear MMSE equalizer $\hat{\boldsymbol{w}}_{d}$ with various decision delays $d \in \mathcal{D}$. The results depicted in Fig.2 agree with the predicted relative BER performance using $\lambda\left(\hat{\boldsymbol{w}}_{d}\right)$.

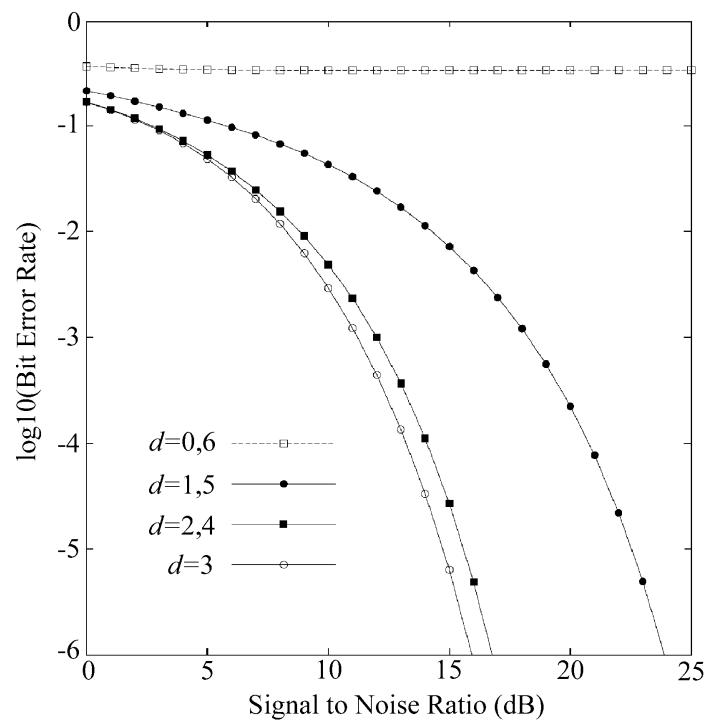

Fig.2 Bit error rate performance as a function of decision delay for the channel $H_{1}(z)=0.66+1.0 z^{-1}-0.66 z^{-2}$ with an equalizer length $N=5$. A MMSE design was employed

For the same SNR of $25 \mathrm{~dB}$, the MBER weight vectors $\tilde{\boldsymbol{w}}_{d}$ for all $d \in \mathcal{D}$ were also calculated numerically using the MBER optimization algorithm given in [8]. The related minimum distance measures $\lambda\left(\tilde{\boldsymbol{w}}_{d}\right)$ for $d=0,1, \cdots, 6$ were

$$
-1.117,0.520,0.899,0.922,0.899,0.520,-1.117 \text {. }
$$

The BER performance of the linear MBER equalizer $\tilde{\boldsymbol{w}}_{d}$ with $d \in \mathcal{D}$ are illustrated in Fig.3. The results shown in Fig.3 agree with the predictions using $\lambda\left(\tilde{\boldsymbol{w}}_{d}\right)$.

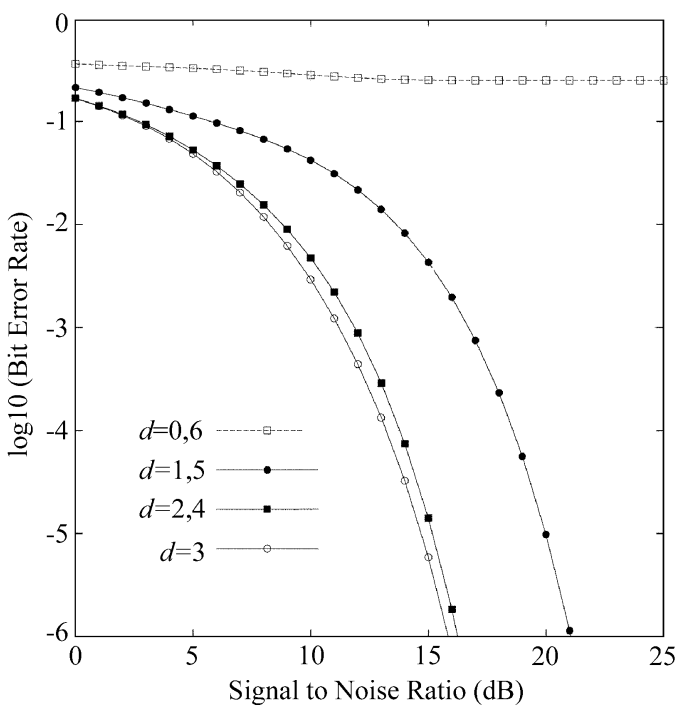

Fig.3 Bit error rate performance as a function of decision delay for the channel $H_{1}(z)=0.66+1.0 z^{-1}-0.66 z^{-2}$ with an equalizer length $N=5$. A MBER design was employed

Also $\lambda\left(\tilde{\boldsymbol{w}}_{1}\right)$ and $\lambda\left(\tilde{\boldsymbol{w}}_{5}\right)$ are significantly larger than $\lambda\left(\hat{\boldsymbol{w}}_{1}\right)$ and $\lambda\left(\hat{\boldsymbol{w}}_{5}\right)$. Inspecting Figs.2 and 3, it can 
be seen that the corresponding MBER equalizers have much better BER performance than the related MMSE equalizers. This further confirms the usefulness of $\lambda\left(\boldsymbol{w}_{d}\right)$ as a relative BER performance indicator.

Example 2. The transfer function of a CIR was given by $H_{2}(z)=0.6996+0.6646 z^{-1}-0.2623 z^{-2}$ and equalizer length chosen as $N=6$. The range of decision delays was therefore $\mathcal{D}=\{0,1, \cdots, 7\}$. Given a channel SNR of $24 \mathrm{~dB}$, the MMSE weight vectors $\hat{\boldsymbol{w}}_{d}(9)$ for each decision delay $d \in \mathcal{D}$ were calculated and the corresponding minimum distance measures $\lambda\left(\hat{\boldsymbol{w}}_{d}\right)$ were found to be

$-0.457,-0.237,0.037,0.217,0.315,0.371,0.141,-0.872$

It can be seen that for the MMSE design $d=0,1,7$ result in nonlinear separable problems with the worst BER performance given by $d=7$. The optimal decision delay is $d=5$, which has the smallest BER. These predictions using $\lambda\left(\hat{\boldsymbol{w}}_{d}\right)$ are confirmed by the actual BER performance depicted in Fig.4.

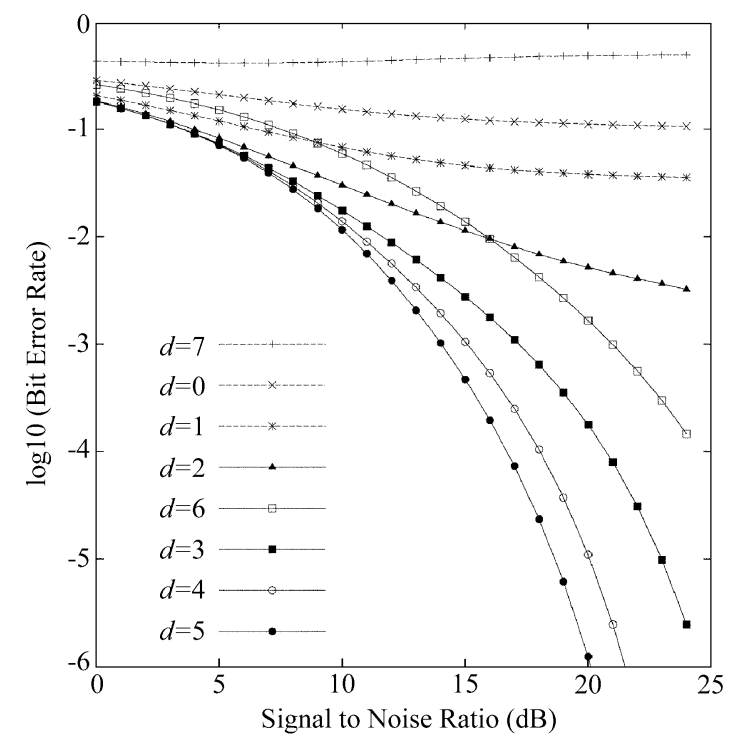

Fig.4 Bit error rate performance as a function of decision delay for the channel $H_{2}(z)=0.6996+0.6646 z^{-1}-$

$0.2623 z^{-2}$ with an equalizer length $N=6$. A MMSE design was employed

The same process was repeated for the MBER design, and the corresponding minimum distance measures $\lambda\left(\tilde{\boldsymbol{w}}_{d}\right)$ were found to be

$$
-0.398,0.136,0.262,0.328,0.364,0.401,0.179,-1.097 \text {. }
$$

It can be seen that for the MBER design only $d=0,7$ result in nonlinear separable problems with the worst BER performance given by $d=7$. The optimal decision delay for the MBER design is also $d=5$. Fig. 5 illustrates the actual BER performance for the linear MBER equalizers $\tilde{\boldsymbol{w}}_{d}$ for $d \in \mathcal{D}$.

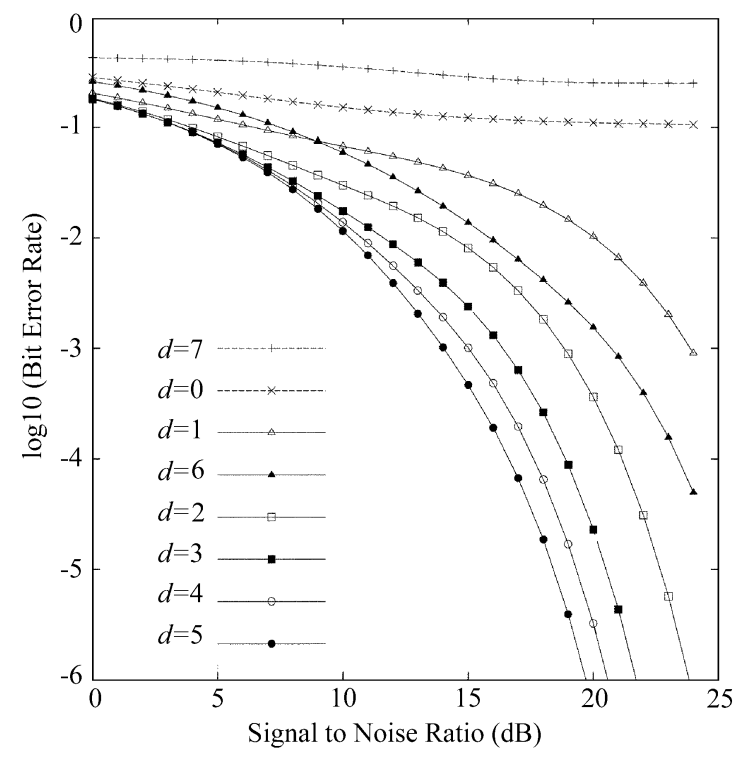

Fig.5 Bit error rate performance as a function of decision delay for the channel $H_{2}(z)=0.6996+0.6646 z^{-1}$ $0.2623 z^{-2}$ with an equalizer length $N=6$. A MBER design was employed

\section{Extension to a general modulation scheme}

We now show how to extend the proposed method to a general modulation scheme. First consider a $G$ level pulse amplitude modulation ( $G$-PAM) scheme, where $s(k)$ is drawn from the symbol set

$$
\left\{s_{l}=2 l-1-G, 1 \leqslant l \leqslant G\right\} .
$$

Therefore

$$
\boldsymbol{s}(k) \in \mathcal{S}=\left\{\boldsymbol{s}_{i}, 1 \leqslant i \leqslant N_{s}\right\}
$$

where $N_{s}=G^{L}$, and

$$
\overline{\boldsymbol{x}}(k) \in \mathcal{X}=\left\{\overline{\boldsymbol{x}}_{i}=H \boldsymbol{s}_{i}, 1 \leqslant i \leqslant N_{s}\right\} .
$$

The set $\mathcal{X}$ can be partitioned into $G$ subsets relative to the value of $s(k-d)$

$$
\mathcal{X}_{l}=\left\{\overline{\boldsymbol{x}}_{i} \in \mathcal{X}: s(k-d)=s_{l}\right\}, 1 \leqslant l \leqslant G .
$$

It can readily be proved that [9] for $1 \leqslant l \leqslant G-1$, $\mathcal{X}_{l+1}$ is a shifted version of $\mathcal{X}_{l}$ by the amount $2 \boldsymbol{h}_{d}$. That is,

$$
\mathcal{X}_{l+1}=\mathcal{X}_{l}+2 \boldsymbol{h}_{d}, 1 \leqslant l \leqslant G-1 .
$$

This shift property enables us to use any two adjacent subsets $\mathcal{X}_{l}$ and $\mathcal{X}_{l+1}$ when considering the degree of linear separability of the equalizer. Specifically, let us choose $l=G / 2$. Then $s_{G / 2}=-1$ and $s_{1+G / 2}=+1$. It is clear that this is equivalent to the BPSK case presented in this paper. 
For a general complex-valued modulation scheme, such as a quadrature amplitude modulation (QAM) scheme, the extension is more involved, but the derivation can be carried out similarly.

\section{Conclusions}

A simple but computationally efficient method has been presented to determine the optimal decision delay parameter that achieves the smallest bit error rate for a given linear equalizer design. The proposed method calculates a minimum distance measure for each feasible decision delay value and chooses the decision delay that achieves the maximum of this minimum distance measure. The usefulness of this technique has been demonstrated using two examples involving both minimum mean square error and minimum bit error rate equalization designs.

\section{References}

[1] S. U. H. Qureshi, Adaptive equalization, Proc. IEEE, vol. 73, no. 9, pp. 1349-1387, 1985.

[2] J. G. Proakis, Digital Communications, 3rd edition, New York, McGraw-Hill, 1995.

[3] E. S. Chng, B. Mulgrew, S. Chen, G. Gibson, Optimum lag and subset selection for a radial basis function equaliser, Proc. 5th IEEE Workshop Neural Networks for Signal Processing (Cambridge, USA), pp. 593-602, Aug.31-Sept.2, 1995.

[4] X. Li, H. H. Fan, Direct blind equalization with best delay by channel output whitening, IEEE Trans. Signal Processing, vol. 49, no. 7, pp. 1556-1563, 2001.

[5] S. Chen, B. Mulgrew, E. S. Chng, G. Gibson, Space translation properties and the minimum-BER linear-combiner DFE, IEE Proc. Communications, vol. 145, no. 5, pp. 316-322, 1998.

[6] B. Mulgrew, S. Chen, Adaptive minimum-BER decision feedback equalisers for binary signalling, Signal Processing, vol. 81, no. 7, pp. 1479-1489, 2001

[7] C. C. Yeh, J. R. Barry, Adaptive minimum bit-error rate equalization for binary signaling, IEEE Trans. Communications, vol. 48 , no. 7 , pp. $1226-1235,2000$
[8] S. Chen, A. K. Samingan, B. Mulgrew, L. Hanzo, Adaptive minimum-BER linear multiuser detection for DS-CDMA signals in multipath channels, IEEE Trans. Signal Processing, vol. 49, no. 6, pp. 1240-1247, 2001.

[9] S. Chen, L. Hanzo, B. Mulgrew, Adaptive minimum symbolerror-rate decision feedback equalization for multilevel pulseamplitude modulation, IEEE Trans. Signal Processing, vol. 52, no. 7, pp. 2092-2101, 2004.

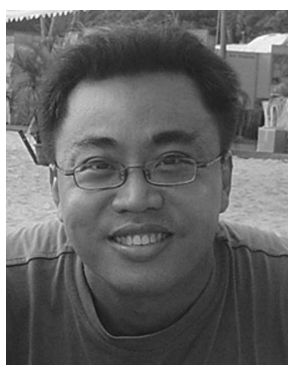

Eng Siong Chng received his university education at the University of Edinburgh, Edinburgh, Scotland (BEng 1991, PhD 1995). After his PhD, he spent 6 months in Japan, working as a researcher for Riken. After working in industry in Singapore for 7 years, he joined the School of Computer Engineering, Nanyang Technological University in 2003. His research interests are in digital signal processing for communication applications, speech and handwriting recognition and noise reduction.

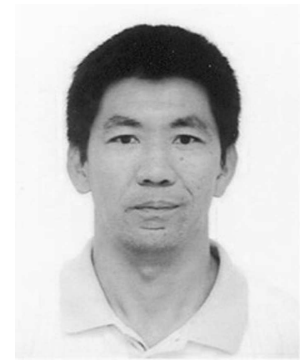

Sheng Chen obtained a BEng degree in control engineering from the East China Petroleum Institute, Dongying, China, in 1982, and a $\mathrm{PhD}$ degree in control engineering from the City University at London in 1986 . He joined the School of Electronics and Computer Science at the University of Southampton in September 1999. He previously held research and academic appointments at the Universities of Sheffield, Edinburgh and Portsmouth. Dr Chen is a Senior Member of the IEEE in the USA. His recent research works include adaptive nonlinear signal processing, modeling and identification of nonlinear systems, neural networks and machine learning, finite-precision digital controller design, evolutionary computation methods and optimization. He has published over 200 research papers. In the database of the world's most highly cited researchers in various disciplines, compiled by the Institute for Scientific Information (ISI) of the USA, Dr Chen is on the list of highly cited researchers in the category that covers all branches of engineering subject, see www.ISIHighlyCited.com. 